\title{
Telepresence System Using High-resolution Omnidirectional Movies and a Reactive Display
}

\author{
Sei IKEDA, Tomokazu SATO, Masayuki KANBARA and Naokazu YOKOYA \\ Vision and Media Computing Laboratory \\ Nara Institute of Science and Technology \\ 8916-5 Takayama, Ikoma, Nara 630-0192, Japan \\ \{sei-i,tomoka-s, kanbara,yokoya\}@is.aist-nara.ac.jp
}

\begin{abstract}
This paper describes a novel telepresence system that uses high-resolution movies and a reactive display system with a treadmill. In this system, users can walk through a virtualized environment by actually walking on a treadmill. According to walking motion which is detected by using 3-D position sensors put on both legs, the virtualized environment captured by an omnidirectional multi-camera system is projected on a multi-screen display.
\end{abstract}

\section{Introduction}

Technology that enables users to experience a remote site virtually is called telepresence. A telepresence system using real environment images is expected to be used in the field of entertainment and medicine. To realize a rich presence, the following functions are needed for the system: high-resolution displayed image, interlock with body motion, free moving position and free viewing direction. However, most existing systems [1,2] lack some of such functions.

In this paper, a novel telepresence system is proposed. The system is equipped with a high-resolution immersive display and a treadmill for input and output devices. High-resolution omnidirectional movies acquired by an omnidirectional multi-camera system [3] are projected to an immersive screen by using detected user's walking motion. Our system realizes almost all of the abovementioned functions except that user's motion is limited to one degree of freedom. The following sections describe the detail of the proposed system.

\section{Proposed Telepresence System}

As shown in Figure 1, the proposed system is composed of (a) a walking motion interface, (b) a graphics PC cluster and (c) an immersive three-screen display. The walking motion interface detects user's walking motion as input data, and sends calculated displacement information to the PC cluster. The PC cluster draws

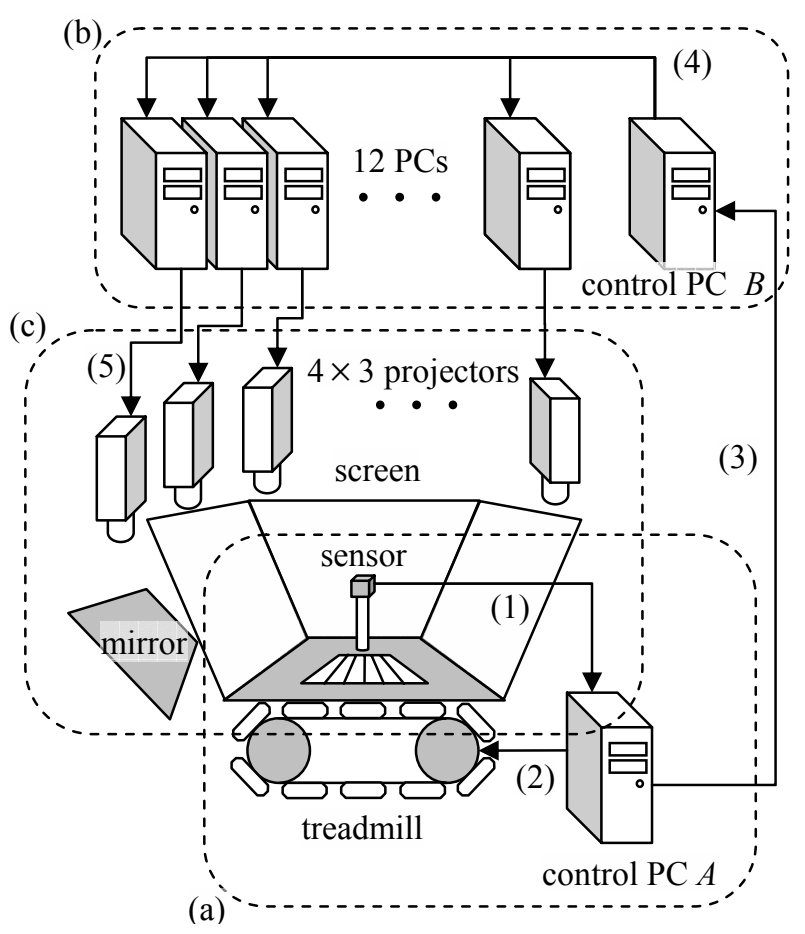

Figure 1. Configuration of the proposed system.

twelve images synchronized with the speed of walking because each screen image is generated by four projectors. As output data, these movies are displayed according to the user's motion. These components are described in some more detail below.

\section{(a) Walking Motion Interface}

This interface is composed of a treadmill (WalkMaster), a couple of 3-D position sensors (Polhemus Fastrak) and PC $A$ (Intel Pentium $42.4 \mathrm{GHz}$ ) for control as illustrated in Figure 1. User's walking motion is detected by two 3$\mathrm{D}$ position sensors fixed on user's legs (Figure 1(1)). The treadmill is controlled by PC $A$ based on position information from the sensors (Figure 1(2)). The belt of the treadmill is automatically rotated so that the center of 
gravity of two sensors coincides with the center of the belt area. Although a user can walk any direction on this device [4], only the forward direction is used for the present telepresence system. PC $A$ calculates displacement of user's position and sends it to the graphics PC cluster (Figure 1(3)).

\section{(b) Graphics PC Cluster}

The graphics PC cluster is composed of twelve PCs (CPU: Intel Pentium $41.8 \mathrm{GHz}$, Graphics Card: Geforce4 Ti4600) and a control PC $B$ (Intel Pentium 4, $1.8 \mathrm{GHz}$ ). Each graphics PC is networked through $100 \mathrm{Mbps}$ LAN and controlled by PC $B$. PC $B$ sends frame indexes to twelve PCs using UDP protocol simultaneously (Figure 1(4)). Each machine draws synchronized frame XGA images according to the user's motion (Figure 1(5)). Note that the images are accumulated in local hard disk in advance and only the frame index is carried via network.

\section{(c) Immersive Display}

The immersive display is composed of three slanted rearprojection screens (Solidray VisualValley) and twelve projectors. To obtain a wide field of view, the screens are located in user's front, left and right sides. To realize high-resolution image projection, each screen image is projected through a mirror by four projectors.

The resolution of each projector is $1024 \times 768(\mathrm{XGA})$ pixels. Because there are some overlapping areas projected by multiple projectors and some areas are not projected on the screen, the resolution of each screen is potentially about 2 million pixels.

\section{Experiment}

The subjective evaluation experiment was conducted using the proposed telepresence system shown in Figure 2. Omnidirectional movies used in this experiment are acquired by an omnidirectional multi-camera system Ladybug mounted on a car moving at an approximately constant speed. Figure 3 shows a frame of input movie for twelve projectors.

We have confirmed that the proposed telepresence system provides us with the feeling of rich presence in remote sites in this experiment. We have also confirmed that geometric discontinuity between regions projected by different projectors and synchronization error could not be recognized. However, some image discontinuities were observed in scene areas very close to the camera system due to its violation of single view point constraint and poor presence was felt due to the limitation of user's motion. We also felt unnatural in the control of the treadmill when a user begins to walk, because the motion of upper part of the body is not considered in motion

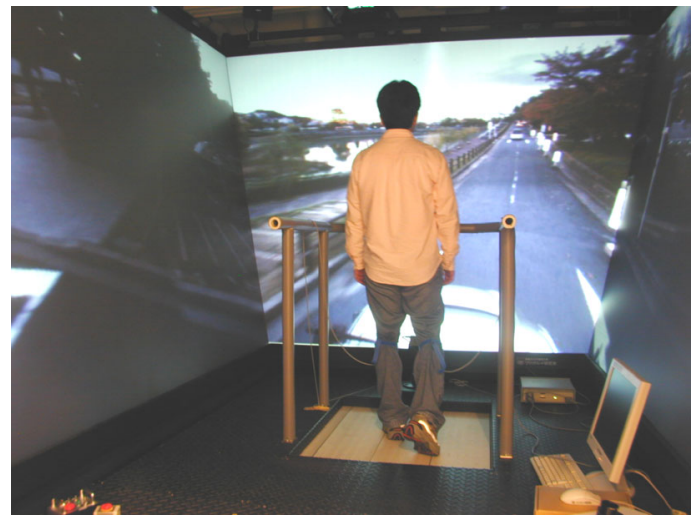

Figure 2. Appearance of telepresence system.

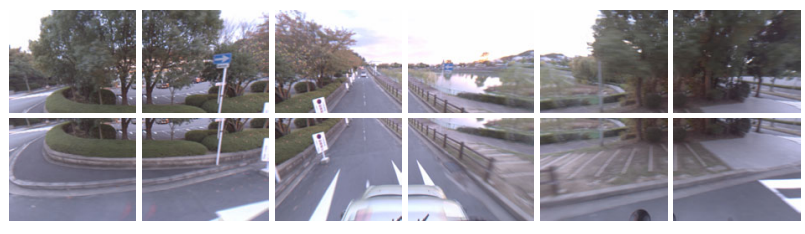

Figure 3. A frame of input movie.

measurement; that is, the displayed image is not synchronized with head motion but with leg motion.

\section{Summary}

In this paper, a novel telepresence system using an immersive display and a treadmill is proposed. This system can interactively present high-resolution real movies according to walking motion. The experiment has shown that the proposed telepresence system provides us with the feeling of rich presence in remote sites.

The following investigations are left as important future work. One is to control the belt rotation of treadmill naturally. The other is to relax the limitation of user's motion.

\section{References}

[1] Y. Onoe et al., "Telepresence by Real-time Viewdependent Image Generation from Omnidirectional Video Streams", Computer Vision and Image Understanding, vol. 71, No. 2, pp. 154-165, 1998.

[2] H. Noma and T. Miyasato, "Design for Locomotion Interface in a Large Scale Virtual Environment, ATLAS: ATR Locomotion Interface for Active Self Motion", Proc. ASME-DSC, vol.64, pp.111-118, 1998.

[3] S. Ikeda et al., "A Calibration Method for an Omnidirectional Multi-camera System", Proc. SPIE, vol. 5006, pp.499-507, 2003.

[4] H. Iwata, "Walking About Virtual Environments on an Infinite Floor", Proc. IEEE Virtual Reality '99, pp. 286-293, 1999. 